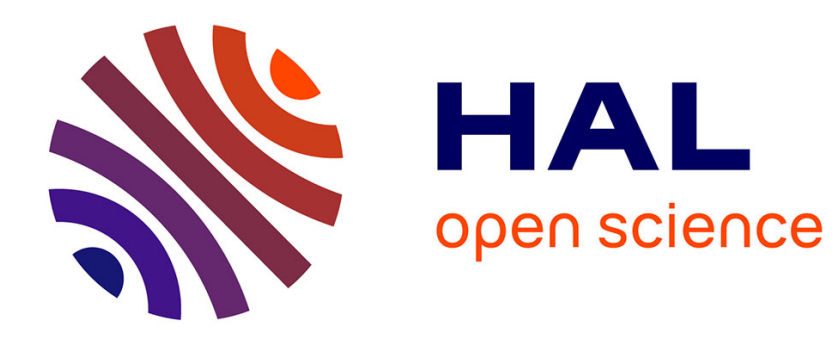

\title{
On the specification for solvers of polynomial systems
}

Daniel Lazard

\section{To cite this version:}

Daniel Lazard. On the specification for solvers of polynomial systems. 5th Asian Symposium on Computer Mathematics - ASCM 2001, Sep 2001, Matsuyama, Japan. pp.66 - 75, 10.1142/9789812799661_0007. inria-00107534

\section{HAL Id: inria-00107534 \\ https://hal.inria.fr/inria-00107534}

Submitted on 19 Oct 2006

HAL is a multi-disciplinary open access archive for the deposit and dissemination of scientific research documents, whether they are published or not. The documents may come from teaching and research institutions in France or abroad, or from public or private research centers.
L'archive ouverte pluridisciplinaire HAL, est destinée au dépôt et à la diffusion de documents scientifiques de niveau recherche, publiés ou non, émanant des établissements d'enseignement et de recherche français ou étrangers, des laboratoires publics ou privés. 


\title{
On the specification for solvers of polynomial systems
}

\author{
Daniel Lazard* \\ LIP6, Université P. et M. Curie, \\ 75252 Paris Cedex 05, France
}

\begin{abstract}
In this paper, the different methods for solving polynomial systems (Gröbner bases, triangular sets, ...) are compared on an example coming from geometry. It is shown that these methods may be combined for automatically studying the number of admissible real solutions of a polynomial system depending on parameters; this study provides as output a description of this number as a function of the parameters.
\end{abstract}

\section{Introduction}

This paper is, in some sense, a continuation of meeting ASCM 2000: It makes explicit the theses of author's paper for this meeting [8] on a problem which is solved in another paper of the same meeting $[9]$.

Many algorithms have been designed for solving polynomial systems, especially through Gröbner bases and triangular or characteristic sets. However, it appears that the output they provide is usually not very well suited for systems of positive dimension which have infinitely many solutions.

The most common case of a systems of positive dimension is a system which depends on parameters and which has a finite number of solutions for almost all values of the parameters. Usually, for such systems, one is interested only in real solutions satisfying some sign conditions.

For such systems, the output which is wanted consists in structural information on the solutions. This may be the number of solutions as a function of the parameters and also some information on the special values of the parameters where the number of solutions varies, which allows one to interpret physically this variation.

In this paper, we describe on a rather simple example what we mean by such a resolution, and we will discuss in which way existing algorithms are, or not, well suited for this purpose.

Our example is the system $\left(f_{1}=0, f_{2}=0, f_{3}=0\right)$, where

$$
\begin{aligned}
& f_{1}=\left(c^{2}-l^{2}\right) y^{2}+\left(b^{2}-l^{2}\right) z^{2}+\left(b^{2}+c^{2}-a^{2}-2 l^{2}\right) y z \\
& f_{2}=\left(a^{2}-l^{2}\right) z^{2}+\left(c^{2}-l^{2}\right) x^{2}+\left(c^{2}+a^{2}-b^{2}-2 l^{2}\right) z x \\
& f_{3}=\left(b^{2}-l^{2}\right) x^{2}+\left(a^{2}-l^{2}\right) y^{2}+\left(a^{2}+b^{2}-c^{2}-2 l^{2}\right) x y
\end{aligned}
$$

This system was introduced by Lu Yang and Zhenbing Zeng [9] for studying the points where the three lines passing through the point and one vertex of a triangle intersect the triangle in three segments of the same length. In their conclusion, they asked for an automatization of their study. We will show that the present knowledge allows a complete automatization of their discussion, even if some general purpose programs yet need to be written or interfaced in order to avoid human intervention.

In the above system, $a, b, c$ are real parameters and represent the lengths of the sides of a triangle $A B C$; thus they are positive and satisfy triangle inequalities. The unknown $l$ is the common length of the intersections and should be real and positive. The three remaining unknowns $x, y, z$ are the homogeneous barycentric coordinates of the unknown point; thus they should be real and have a non zero sum; moreover, they have to be viewed as projective coordinates, i.e. $(x, y, z)$ and $(\lambda x, \lambda y, \lambda z)$ define the same point and are viewed as equal.

Notations and terminology: In this paper we strongly use triangular systems and the various algorithms for computing them. As the terminology in this area is not yet fixed, we have either to redefine

*E-mail: Daniel.Lazard@lip6.fr 
each notion or to choose the terminology of a published paper. For brevity, we have taken the second option, and we will use without recalling it the terminology of the paper by Aubry et al. [2].

\section{Decomposition of the system}

The first thing to do with a system like our example consists in decomposing the space of solutions into irreducible components.

In most systems coming from applications, there is only one component and the step is vacuous.

However, in some cases, some irrelevant components are introduced by the choice of the equations modeling the problem. In some other cases, the set of admissible solutions is not irreducible, which means that there are solutions of different physical nature. When this is the case, it is important that the output provides information on such a phenomenon. In our example, both cases occur: irrelevant components and solutions of different nature.

Decomposing an algebraic set into components is equivalent to decomposing the radical of its defining ideal into prime ideals. This may be done, in principle, by any algorithm which decomposes the set of zeros into triangular systems $[13,12,6]$. But, for many of these algorithms, one needs, at the end, to remove components which are included in the adherence (for Zariski or usual topology) of others, and this operation may need computation of Gröbner bases.

However, our example, as it is rather usual, is a complete intersection, and removing extraneous components is very easy: A well known theorem of algebraic geometry asserts that any prime or primary component has a co-dimension which is at most equal to the number of equations. Thus, for getting a prime decomposition by triangular sets, one may stop the computation when one knows that the triangular sets which remain to be computed have more elements than the initial system. Moreover, if they have the same dimension, the mutual inclusions may be easily tested by pseudo-division, and, for most algorithms, such a test is not even necessary, because they provide directly irredundant decomposition for components of maximal dimension.

Thus, in our example, all prime components have characteristic sets of 3 elements, and, after a factorization, any algorithm computing triangular sets provide a prime decomposition. However the quality (i.e. the size and the readability) strongly depends on the algorithms, as we will see below.

For this purpose, we fix the order on the variables as $x>y>z>l>a>b>c$, and we look on the various possible outputs.

The original $\mathrm{Wu}$ Wen-tsün algorithm, for example, provides a first triangular set containing the product of a power of $z$ by a polynomial in $l, a, b, c$, and also a polynomial with an initial which is a multiple of $z$. Thus this triangular set has no regular zero and should simply be removed.

For this reason, we will limit ourselves, from now on, to algorithms which produce regular sets, and even separable regular sets, which are square-free sets in the terminology of [7], or equivalently regular sets for which the derivatives of their elements with respect of their main variables (their separants) are not zero-divisors modulo the saturated ideal.

Such algorithms provide usually the following output (this may depends on various parameters, especially on the square-free algorithm which is used);

$$
\begin{aligned}
& (z, y, x) \\
& \left(l, \quad c^{2} y^{2}+b^{2} z^{2}+\left(b^{2}+c^{2}-a^{2}\right) y z\right. \\
& \left.\quad\left(y c^{4}-y a^{2} c^{2}-y b^{2} c^{2}-z b^{4}+z a^{2} b^{2}+z b^{2} c^{2}\right) x+z^{2} a^{2} b^{2}-y^{2} a^{2} c^{2}\right) \\
& \left(l^{2}-a^{2}, \quad\left(c^{2}-a^{2}\right) y^{2}+\left(b^{2}-a^{2}\right) z^{2}+\left(b^{2}+c^{2}-3 a^{2}\right) y z, \quad x\right) \\
& \left(l^{2}-b^{2}, \quad y, \quad\left(c^{2}-b^{2}\right) x^{2}+\left(c^{2}+a^{2}-3 b^{2}\right) z x+\left(a^{2}-b^{2}\right) z^{2}\right) \\
& \left(l^{2}-c^{2}, \quad z, \quad\left(b^{2}-c^{2}\right) x^{2}+\left(a^{2}+b^{2}-3 c^{2}\right) x y+\left(a^{2}-c^{2}\right) y^{2}\right) \\
& \left(h, \quad f_{1}, \quad\left(b^{2}-l^{2}\right) f_{2}-\left(c^{2}-l^{2}\right) f_{3}\right)
\end{aligned}
$$

where $h$ is the (irreducible) polynomial

$$
h:=16 l^{4}-8\left(a^{2}+b^{2}+c^{2}\right) l^{2}+3(a+b+c)(a+b-c)(b+c-a)(c+a-b),
$$

and in which the third element of (7) is linear in $x$, with a coefficient of $x$ containing 14 terms.

With this output, it is easy to get the prime decomposition: It suffices to replace, in triangular sets (4), (5) and (6), the first element by both of its factors. This gives 9 irreducible components. It is easy 
to prove that they are effectively irreducible: For (2), its immediate; for (3), it suffices to verify that the second element is irreducible, the third one being linear in its main variable; for (4), (5) and (6), it suffices also to verify that the element which is quadratic in its main variable is irreducible; finally, for (7) only, non-trivial computation is needed: We have to prove that $h$ is irreducible and that the second element is irreducible in the algebraic function field defined by $h$.

Thus the above output is rather convenient from the point of view of the prime components. However for getting easily the solutions of the problem, some initials are too involved and give a set of regular zeros which is too small. In fact, if one normalizes these triangular sets in the meaning of [7], it appears that some content may be removed from the third elements of (3) and (7); but this normalization replaces the second element of (7) by a much bigger element. If normalization is only done when it allows one to remove a content, then only (3) and (7) are changed and become:

$$
\begin{aligned}
& \left(l, \quad c^{2} y^{2}+b^{2}-z^{2}+\left(b^{2}+c^{2}-a^{2}\right) y z, \quad x+y+z\right) \\
& \left(h, \quad f_{1},\right. \\
& \left.\quad 2 x\left(b^{2}-c^{2}\right)+y\left(4 l^{2}-3 a^{2}+b^{2}-c^{2}\right)-z\left(4 l^{2}-3 a^{2}-b^{2}+c^{2}\right)\right)
\end{aligned}
$$

With these new triangular sets, it appears that the saturated ideals (and thus the prime ideals) are generated by the triangular sets, except for the last component (9). For this component, the zeros which are not regular satisfy $b^{2}=c^{2}$; by symmetry of the problem, the zeros which are not regular for any ordering on $x, y, z$ satisfy $a^{2}=b^{2}=c^{2}$, and thus correspond to the equilateral triangle.

Remark 1 From the above discussion, we may extract a conclusion, which seems of general value: When there are several irreducible components, prime decomposition provides useful information. The description of prime ideals which appears to be the most compact one and the most useful one consists in regular triangular sets in which polynomials are normalized only if this operation removes some contents.

\section{Primary decomposition}

It is not really useful here, but, in some case one may also need the complete primary decomposition, and not only the prime decomposition. When one knows that there are no embedded components, this may be done rather easily from the prime decomposition, by localization. This is clearly the case for our example, because it is complete intersection (the number of equations is equal to the co-dimension of the ideal).

More precisely, given a prime component $\mathcal{P}$, let $S$ be a set of polynomials not contained in $\mathcal{P}$, such that any other prime component contains some element of $S$; the primary component associated to $\mathcal{P}$ may be obtained by saturating the ideal to be decomposed by the elements of $S$. We will explicitly describe below this operation for the prime component $(x, y, z)$, which is the only one for which the result is not easy. In fact, for the prime ideals defined by (4), (5), (6) and (9), the primary component is the same as the prime component. For (8), the primary component is simply obtained by replacing $l$ with $l^{2}$ in (8).

For the component $(x, y, z)$, the above set $S$ may be $\left[l, l^{2}-a^{2}, l^{2}-b^{2}, l^{2}-c^{2}, h\right]$. Then a Gröbner basis of the primary component may be computed by the following program (written in Maple) where $w$ is an auxiliary variable and order a monomial ordering eliminating $w$, for example a lexicographical ordering with $w$ as biggest variable. In this program, the map(primpart ...) operations are optional but may improve efficiency.

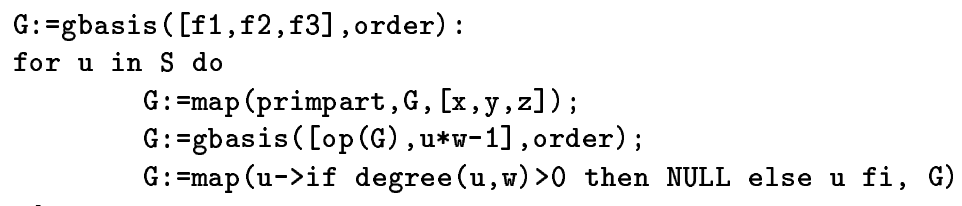

The resulting Gröbner basis contains about 30 polynomials (this depends on the ordering which is chosen), among which appear all the 15 monomials of degree 4 in $x, y, z$. In fact, it may be easily deduced without any computation from multivariate resultant theory that the primary component is generated by the input polynomials $f_{1}, f_{2}, f_{3}$ (of degree 2 in $x, y, z$ ), the monomials of degree 4 in $x, y, z$, and some polynomials of degree 3 in $x, y, z$. 
It is interesting to note that the very simple prime component $(x, y, z)$ has a complicated primary component, the Gröbner basis of which contains many polynomials depending on $l, a, b, c$, some of them requiring several lines for being written down.

Remark 2 It is well known that primary decompositions are usually difficult to compute. This example shows also that primary decompositions would hardly be useful without a compact way to describe primary ideals (as characteristic sets are for prime ideals).

\section{Admissible solutions: Critical loci}

When we have the prime components, we have to discuss the number of admissible solutions as a function of the parameters. Let us recall that the conditions on the solutions are: $a, b, c, l$ all positive, $x+y+z \neq 0$ and $a+b-c, b+c-a, c+a-b$ all positive, or equivalently Degen positive, where

$$
\text { Degen }=(a+b+c)(a+b-c)(b+c-a)(c+a-b)=2 a^{2} b^{2}+2 b^{2} c^{2}+2 c^{2} a^{2}-a^{4}-b^{4}-c^{4} .
$$

The second condition shows that we only need to consider the components (4), (5), (6) and (9). In their paper, Lu Yang and Zhenbing Zeng use several tricks for this discussion; we will show that this discussion may be completed in a purely automatic way without any special trick.

The discussion may be done on the triangular systems as they are provided in preceding sections, and we have done it. However, the fact that the values we are looking for $x, y, z$ lie in a projective space, introduce some technical difficulties.

Therefore, it is better to de-homogenize the system by adding the equation $x+y+z-1=0$. This is very natural, because it consists in replacing homogeneous barycentric coordinates by actual barycentric coordinates.

Remark 3 Some reader may wonder why this has not been done from the beginning. There are several reasons for this. First we wanted to keep, as much as possible, the system as it was provided by the Chinese authors. Secondly, de-homogenizing from the beginning would throw away the two components (2) and (8), and all remaining primary components would be prime. Finally, the decomposition into triangular sets is much easier for the system provided by Lu Yang and Zhenbing Zeng: For Aubry's software in Axiom [1], computating time passes from 3 seconds to more than 800 seconds when equation $x+y+z=1$ is added, and most other software fail on such a difficult system.

The reason for this higher complexity seems to lie in the following: the initial homogeneous system consists of three polynomials, each of them depending only on two among $x, y, z$. Eliminating these variables for getting a polynomial in $l, a, b, c$ is easy by using usual resultant (as the Chinese authors did) or, almost equivalently, by pseudo-division. On the other hand, replacing, says, $x$ by $1-y-z$ provides three dense bivariate polynomials in $y, z$, and elimination is much more difficult in this context, without using Gröbner bases.

However, this higher complexity of the non-homogeneous problem may be an artefact of the algorithms which are used: The experimental algorithm Fr of Faugère (http://calfor.lip6.fr/ jcf) computes the Gröbner bases of all irreducible components in 0.3 seconds in the non-homogeneous case and in 0.2 seconds in the homogeneous case, on a Pentium III, $800 \mathrm{Mh}$.

Putting $x=1-y-z$ in (4), (5), (6) and (9), it is easy to compute regular triangular sets which are characteristic sets of corresponding ideals. We obtain

$$
\begin{aligned}
T_{a}:= & \left(l^{2}-a^{2}, \quad a^{2} z^{2}+\left(b^{2}-c^{2}-a^{2}\right) z+c^{2}-a^{2}, \quad 1-y-z\right) \\
T_{b}:= & \left(l^{2}-b^{2}, \quad b^{2} z^{2}+\left(a^{2}-b^{2}-c^{2}\right) z+c^{2}-b^{2}, \quad y\right) \\
T_{c}:= & \left(l^{2}-c^{2}, \quad z, \quad c^{2} y^{2}+\left(a^{2}-b^{2}-c^{2}\right) y+b^{2}-c^{2}\right) \\
T_{g}:= & \left(h, \quad 3 l^{2} z^{2}-2 l^{2} z-l^{2}+c^{2}\right. \\
& \left.\quad\left(4 l^{2}-3\left(a^{2}+c^{2}-b^{2}\right)\right) y-\left(4 l^{2}-3\left(a^{2}+b^{2}-c^{2}\right)\right) z\right)
\end{aligned}
$$

A very simple idea for discussing the number of admissible solutions consists in letting the parameters $a, b, c$ vary and looking on the values of them for which the number of admissible solutions do change. In fact, this number varies if some inequality condition becomes zero, or if some solution passes through infinity, or if some Jacobian vanishes.

Let us describe in more detail on this example how to compute these loci where the number of admissible solutions may change. We will do this through two approaches, by Gröbner bases computations 
or directly from triangular sets, in order to compare them. In a future paper, we will describe in a more general setting how to compute these loci and will precise the conditions under which such a discussion may apply.

\section{Gröbner bases approach}

Degeneracy conditions: The conditions $a, b, c$ positive and Degen $>0$ simply limit the part of the space of parameters which has to be studied, and no further computation is needed here.

If we put $l=0$ in $T_{a}, T_{b}, T_{c}$ and $T_{g}$, we get $a^{2}, b^{2}, c^{2}$ or Degen (constant term of $h$ ) equal to 0 , showing that this degeneracy condition may only occur outside of the domain of study.

The condition $x+y+z \neq 0$ has been forced by putting $x=1-y-z$.

Solutions at infinity: There may be solutions at infinity only when, for some variable, all polynomials of the lexicographical Gröbner basis, which have this variable as main variable, have an initial which vanishes.

For $T_{a}$, the only initial which is not constant is $a^{2}$. Thus there may be solutions at infinity only for a degenerated triangle. The same is true for $T_{b}$ and $T_{c}$.

For $T_{g}$, the polynomial $h$ having a constant initial, $l$ may not be infinite. The second polynomial of $T_{g}$ has $l$ as initial, which is null only in degenerate cases. For $y$ we have to compute the Gröbner basis, which contains 4 polynomials with $y$ as main variable. Among them, we have the initials $3 c$ and $8 l-6 c$, which may both vanish only for $l=0$.

Thus none of the components has solutions at infinity outside the degenerate cases.

Critical curves: The number of real solutions may change at points where the theorem of implicit functions does not allow to express $y, z, l$ as functions of $a, b, c$. This may be computed by adding to the ideal defining each component the $3 \times 3$ determinants of the Jacobian matrix of the generators of the ideal, with respect to $y, z, l$; then a Gröbner basis computation eliminating $y, z, l$ produces the critical locus which is usually (but not always) an hyper-surface.

For $T_{a}, T_{b}$ and $T_{c}$, the ideal is generated by the triangular set. Thus, there is only one Jacobian matrix to compute and it is triangular. Its determinant is $2 l$ times the derivative of the polynomial which is quadratic in $y$ or $z$. Eliminating $y, z, l$ from $T_{a}$ and both factors (through Gröbner bases), we get $a^{2}$ (degeneracy corresponding to $l=0$ ) and

$$
C_{a}:=4 a^{4}-\text { Degen }=5 a^{4}+b^{4}+c^{4}-2 a^{2} b^{2}-2 b^{2} c^{2}-2 c^{2} a^{2},
$$

and similarly for $T_{b}$ and $T_{c}$.

For $T_{g}$, we have to compute first a Gröbner base of the saturated ideal, consisting of seven polynomials, and then the 35 Jacobian determinants. Eliminating $y, z, l$ from all these polynomials we get 3 polynomials which are the product of

$$
\text { Centre }:=a^{4}+b^{4}+c^{4}-a^{2} b^{2}-b^{2} c^{2}-c^{2} a^{2}=\frac{1}{2}\left(\left(a^{2}-b^{2}\right)^{2}+\left(b^{2}-c^{2}\right)^{2}+\left(c^{2}-a^{2}\right)^{2}\right)
$$

by $a^{2}, b^{2}$ and $c^{2}$, respectively. Let us remark that, if we had taken $l^{2}$ as variable, instead of $l$, we would have obtained only one polynomial, equal to Centre.

Intersections of components: The number of solutions may also change when two components intersect.

The components $T_{a}$ and $T_{b}$ intersect if $l^{2}=a^{2}=b^{2}$ and $x=y=z-1=0$, given the condition $a^{2}=b^{2}$, and similarly for the intersection of $T_{a}$ and $T_{c}$ or of $T_{b}$ and $T_{c}$.

The intersection of components $T_{a}$ and $T_{g}$ may be obtained in substituting $l^{2}$ by $a^{2}$ and $y$ by $1-z$ in $T_{g}$. Putting the resulting ideal in triangular form (or computing its Gröbner basis), we get

$$
\text { Inter }_{a}:=-5 a^{4}+3 b^{4}+3 c^{4}+2 a^{2} b^{2}+2 a^{2} c^{2}-6 b^{2} c^{2}
$$

and one (or two) polynomial which is linear in $y$.

This shows that the two components have exactly one solution in common when Inter $_{a}=0$, and similarly for the other intersections.

\section{Triangular sets approach}

Even for this simple example Gröbner basis computations may be rather heavy, especially for computing critical curves. These Gröbner basis computations may be avoided by using triangular sets. 
In fact, the Jacobian matrix of a triangular set is triangular, and its determinant is the product of the separants, i.e. the derivatives of the polynomials with respect of their main variables. Thus we may split the computation by considering separately the separants.

However, proceeding like this, we get, together in the output, the critical locus, the solutions at infinity and some extraneous locus depending on the ordering of the variables or on the fact that the component is not necessarily complete intersection. Thus we may get irrelevant components which may be removed only by a further study.

In our example, for components $T_{a}, T_{b}$ and $T_{c}$, the characteristic set generates the ideal and both approaches collapse.

For the component $T_{g}$, we get Centre by eliminating $l^{2}$ from $h$ and its separant.

The second element of $T_{g}$ and its separant generate $l^{2}\left(3 c^{2}-4 l^{2}\right)$. Eliminating $l$ from this polynomial and $h$, we get $\left(b^{2}-c^{2}\right)^{2}$ Degen. Eliminating $l^{2}$ from $h$ and the separant (which is also it initial) of the third element of $T_{g}$, we get $\left(a^{2}-b^{2}\right)^{2}$.

Thus, we may conclude that the critical locus is a subset of the union of the zeros of $a^{2}-b^{2}, b^{2}-c^{2}$, Degen and Centre. By permuting the variables, we see immediately that one may replace the first factors by the ideal $\left(b^{2}-c^{2}, c^{2}-a^{2}, a^{2}-b^{2}\right)$. As Centre is in this ideal, the critical locus is contained in the union of the zeros of Degen and Centre.

Thus, using triangular sets, we get (without extra reasoning) a critical set which is too big. It could also miss some critical components, if they were contained in the zeros of the initials. Thus we should also add to the locus the zeros of the initials, which contains also the case of solutions at infinity.

In our example, all these extra loci are relevant for the discussion in following section. But it would not be the case if one were only interested in the solutions of $T_{g}$. If we had not removed contents (by normalization) in triangular sets, we would have many more extra components which would make the computation problematic as it was the case in the paper by Lu Yang and Zhenbing Zeng.

Remark 4 From the preceding considerations, we may conclude that Gröbner basis computation may be heavier for finding the critical loci, but it provides a better solution, consisting only of the relevant loci.

\section{Admissible solutions: The discussion}

In preceding section, we got 10 polynomials such that, if the number of solutions changes (when $a, b, c$ varies), some of these polynomials vanishes.

Thus, for having a complete discussion on the number of solutions as a function of $a, b, c$, it suffices to determine the regions defined by these polynomials, to choose a sample value of $(a, b, c)$ in each of these regions and to count the number of admissible solutions for each of these sample values.

Such a job is exactly what cylindrical algebraic decomposition [3] is supposed to do.

However, things are much easier here because the parameters lie in a projective space of dimension 2 : changing the length unit is equivalent to multipling $a, b, c$ by a constant. Thus we may fix this length unit in order that $a=1$ or $a+b+c=1$ (for example). When this is done, we have to study the regions defined by 10 curves in an affine plane. This may be done automatically by any software for drawing plane curves with a certified topology (for example [5]).

As we are in a rather simple case, it suffices here to draw the curves and to look at the regions they define. We have chosen two ways for representing the space of the parameters $a, b, c$.

The first one (Fig. 1) consists in fixing the vertices $B$ and $C$ of the triangle as the points of coordinates $(1,0)$ and $(-1,0)$ (i.e. $a=2)$. Then, the triangle is represented by the position of vertex $A$ in the upper half plane, i.e., if the triangle is represented by the point of coordinates $(u, v)$, then $b^{2}=v^{2}+(u+1)^{2}$ and $c^{2}=v^{2}+(u-1)^{2}$. This representation has the advantage to show directly the shape of the triangle represented by a point, but it breaks the symmetry of the problem.

The second representation (Fig. 2) consists in representing the triangle $(a, b, c)$ by the point of barycentric coordinates $\left(\frac{a^{2}}{a^{2}+b^{2}+c^{2}}, \frac{b^{2}}{a^{2}+b^{2}+c^{2}}, \frac{c^{2}}{a^{2}+b^{2}+c^{2}}\right)$, relatively to the vertices of an equilateral triangle with vertices of coordinates $(0,2),(\sqrt{3},-1)$ and $(-\sqrt{3},-1)$. The advantage of this is not to break the symmetry and also that all curves are straight lines or conics. We have represented the values for $\left(a^{2}, b^{2}, c^{2}\right)$ for the extremities of the drawn diameters.

The curves computed in the preceding section appear in Fig. 1 and 2, corresponding to both representations: The domain of non-degenerate triangles is the upper half plane in the first representation and the open disk in the second one. The region of triangles such that $a \geq b \geq c$ is delimited by thick lines. The dashed lines correspond to the right-angled triangles. The isoceles triangles $(a=b$ or $b=c$ or $c=a)$ are the big half circles in Fig. 1 and the diameters in Fig. 2. The critical curves $C_{a}=0, C_{b}=0$ 

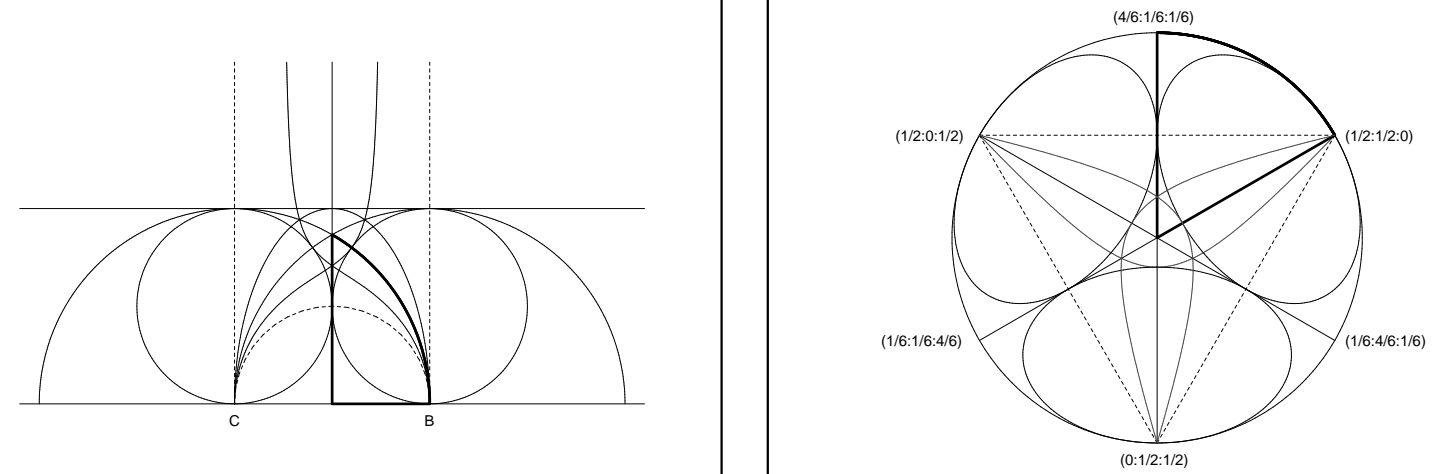

Figure 1: Triangle represented by the position of $A$.

Figure 2: Triangle represented by $\left(a^{2}, b^{2}, c^{2}\right)$ as barycentric coordinates.

and $C_{c}=0$ appear as two small circles and one horizontal straight line in Fig. 1 and as ellipses in Fig. 2. The curves Inter $_{a}=0$, Inter $_{b}=0$ and Inter $_{c}=0$ are the half ellipse and the cubic lines in Fig. 1 and the branches of hyperbolae in Fig. 2. Note that the critical curve of $T_{g}$, which has Centre $=0$ as equation, has only one real point $(a=b=c)$ and does not need to be drawn.

We summarize now as two propositions the results which may be obtained by considering these figures and computing some sample points (in fact, only very few sample points are really needed).

We recall here that $a, b, c$ are the lengths of the edges of a triangle $A B C$ and that the solutions $(x, y, z, l)$ of the studied system represent a point $P$ (called hereafter solution point) of barycentric coordinates $(x, y, z)$ relatively to $A, B, C$, such the three segments which are defined below have the same length $l$. These segments are those which are delimited on lines $P A, P B$ and $P C$ by the corresponding vertex and its opposite edge.

The first proposition describes the number of solutions of each components and their position inside or outside the triangle; the second describes their total number as a function of the parameters $a, b, c$.

Proposition 1 For a non-degenerate triangle $A B C$ with side lengths of $a=B C, b=A C$ and $c=A B$, we have:

- Component $T_{a}$ has two real solutions on side $B C$ (i.e. $x=0$ ) if $C_{a}<0$, one if $C_{a}=0$, and none if $C_{a}>0$. The two latter cases may occur only if a is the smallest side.

If $a$ is the biggest side of the triangle, then $B$ and $C$ lie between the two solution points of $T_{a}$. If $b>a>c$, one of the solution points of $C_{a}$ is inside segment $B C$ and the other is outside, on $B$ side (i.e. $B$ lies between the solution points). If a is the smallest side and $C_{a}<0$, then the solution points lie between $B$ and $C$ if both angles $B$ and $C$ are acute, and lie outside segment $B C$ on the side of the obtuse angle, if any.

- Component $T_{g}$ has two solutions except when the triangle is equilateral $(a=b=c)$, where there is only one multiple solution at center of the triangle. The value of $l$ is the same for both solutions.

If Inter ${ }_{a}$, Inter $_{b}$ and Inter I $_{c}$ are all negative, then both solution points are inside the triangle. If Inter $r_{a}>0$, then one of the solution points is outside side $B C$ of the triangle but inside angle $A$. If Inter $r_{a}=0$, then one of the solutions points lies on segment BC. If two among Inter ${ }_{a}$, Inter $r_{b}$, Inter $_{c}$ are non-negative, the preceding rules apply separately on the two points. At least one among Inter $_{a}$, Inter $_{b}$, Inter $r_{c}$ is negative.

Proof: When the triangle varies, a solution point crosses line $B C$ (says) iff the solution becomes a common solution with $T_{a}$. Thus the position of the solutions with respect to the sides of the triangle does not vary inside the open domains which appear in the figures. On the other hand, when the point representing the triangle crosses one of the curves, exactly one of the solution points crosses the corresponding side of the triangle. It follows that all assertions may be deduced from very few sample points.

Proposition 2 If, without loss of generality, the triangle $A B C$ satisfies $a \geq b \geq c$, the total number of solutions of $T_{a}, T_{g}, T_{c}, T_{g}$ may take the following values (22 different cases), the position of the solution points being defined in the preceding proposition. 
- There are 8 solutions for triangles lying on the 4 connected domains defined by $\left(a>b>c\right.$, Inter $r_{c}<$ $0),\left(\right.$ Inter $_{c}>0$, Inter $\left._{b}<0, C_{c}>0\right),\left(b>c\right.$, Inter $\left._{b}>0, C_{c}>0, a^{2}<b^{2}+c^{2}\right)$ and $\left(b>c, C_{c}>0, a^{2}>\right.$ $\left.b^{2}+c^{2}\right)$.

- There are 6 solutions for triangles lying on the 2 connected domains defined by $\left(C_{c}<0\right.$, Inter $\left.r_{b}>0\right)$ and $\left(C_{c}<0\right.$, Inter $\left._{b}<0, a>b\right)$.

- There are 7 solutions when the triangle lies on the 10 connected arcs defined by $\left(b=c, a^{2}>b^{2}+c^{2}\right)$, $\left(b=c, a^{2}<b^{2}+c^{2}<3 a^{2} / 2\right),\left(3 a^{2} / 4<b^{2}=c^{2}<a^{2}\right),\left(c^{2}<a^{2}=b^{2}<5 c^{2} / 4\right),\left(a^{2}=b^{2}>5 c^{2} / 4\right)$, $\left(\right.$ Inter $\left._{c}=0, a>b>c\right),\left(\right.$ Inter $\left._{b}=0, b>c, C_{c}>0\right),\left(C_{c}=0, a^{2}>b^{2}+c^{2}\right),\left(C_{c}=0, a^{2}<\right.$ $b^{2}+c^{2}$, Inter $\left._{b}<0\right)$ and $\left(C_{c}=0\right.$, Inter $\left._{b}>0, a>b\right)$.

- There are 5 solutions when the triangle lies on the arc defined by $\left(\right.$ Inter $\left._{b}=0, C_{c}<0, a>b\right)$.

- There are 4 solutions when $a=b=c$ (equilateral triangle): The center of the triangle, which is a multiple solution of $T_{g}$, with $l^{2}=3 a^{2} / 4$ and the 3 vertices which are common solutions of 2 components (for example $A$ is a common solution of $T_{b}$ and $T_{c}$ ); these solutions satisfy $l=a$.

- There are 5 solutions if $a^{2}=2 b^{2}=2 c^{2}$ (right-angled isoceles triangle): $A$ is the unique solution of both $T_{b}$ and $T_{c}$, the two solutions of $T_{a}$ on the line $B C$ are symmetric outside the segment $B C$, and the two solutions of $T_{g}$ lie outside the sides $A B$ (for one solution) and $A C$ (for the other).

- There are also 5 solutions for the 2 triangles satisfying $\left(b^{2}=c^{2}=3 a^{2} / 4\right)$ (and thus Inter $_{b}=$ Inter $_{c}=0$ ) or $\left(a^{2}=b^{2}=5 c^{2} / 4\right)$ (and thus $C_{c}=$ Inter $_{c}=0$ ).

- There are 6 solutions for the non degenerate triangle satisfying Inter $r_{b}=C_{c}=0$.

Proof: We know from the preceding proposition the number of solutions of each component for any triangle, and from the preceding section that, when two components intersect, they have exactly one solution in common. Thus, it suffices to enumerate the regions delimited by the curves described in the preceding section and drawn in the figures, and to count how many components intersect in each region.

Remark 5 It should be noted that if we had not supposed that $a \geq b \geq c$, we would have to consider 103 different cases. This is a rather high number for our simple problem. It is thus important to design the output of all the computational steps, in order to avoid, as far as possible to generate more regions than it is necessary.

Doing this, we have got, as byproduct, some information which allows us to limit dramatically the number of sample points which are needed. In fact, 2 points in open regions and 3 special points are sufficient, the latter corresponding to the equilateral triangle and to 2 points where two curves are tangent.

We have the feeling that the computational cost of a better designed output for each step is usually offset by the gain in the final result due to fewer regions and fewer sample points.

Remark 6 It has also to be noted that, besides the number of solutions, we have obtained their topology for free (in this case, the "topology" consists in the description of the position of the solutions with respect to the sides of the triangle).

We guess that this situation is quiet general, and that, for most problems, the topology of the solutions may change only when one crosses a critical curve (when the parameters vary).

\section{Conclusion}

In this paper, we have shown, on a rather small example, that it is possible to automatize the discussion of the number of solutions of a system of equations and inequalities depending on parameters, if the system of equations has a finite number of solutions for almost all values of the parameters.

Such an automatic discussion may, clearly, not provide any information on the mathematical meaning of the solution. In our case, it may be proved, without any algebraic computation, that the solutions of $T_{a}$ are the intersections of the side $B C$ with the circle of radius $a$ centered at $A$, and that the solutions of $T_{g}$ are the foci of the smallest circumscribed ellipse of the triangle (a circle if the triangle is equilateral). Such an interpretation is clearly out of the scope of an automatic computation. On the other hand, we do not know any way to prove that there are no further solutions, other than to do computations like ours.

In a future paper, we will state precisely under which conditions, the algorithm which we have sketched may apply. In the same paper, we will apply it to a problem of celestial mechanics which shows the efficiency of this approach and its limit: the critical curve where the number of solutions changes is defined by a bivariate polynomial which needs more than 10 Mbytes to be stored. The computation 
of this polynomial and of the topology of the corresponding curve need more than a week of CPU time with the most efficient software prototypes.

In practice, this algorithm is limited to problems with only two parameters (or, like here, parameters lying in a projective space of dimension 2). There are several reasons for this, the most evident being the difficulty to represent hyper-surfaces in a space of dimension greather than 2 and to describe the topology they define.

It should also be noticed that the problem of determining the number of solutions as a function of the parameters is a special instance of quantifier elimination. However, quantifier elimination is intractable for problems like the one we have considered here, being doubly exponential in the number of variables and parameters. In fact, we have reduced the problem to one of quantifier elimination in dimension 2 , but, even in this case, direct application of Collins CAD would be almost unfeasible, because it generates many more regions than necessary.

Note that our method is more a meta-algorithm than an algorithm, involving almost all methods or algorithms designed for polynomial system solving. In fact, we need mathematical algorithms for prime decomposition, for saturation (or localization with respect to an element), for elimination, for solving zero-dimensional polynomial systems, for drawing plane curves defined by an implicit equation, and for computing their topology. In turn, these algorithms need, with the present technology, triangular or characteristic sets, Gröbner bases, real solving of univariate polynomials and of polynomial systems.

The fact that, with our method, very difficult examples are tractable lies in the strong recent progresses in all these algorithms $[4,11,10]$.

\section{References}

[1] Aubry, P., and Moreno Maza, M. Triangular sets for solving polynomial systems: a comparative implementation of four methods. Journal of Symbolic Computation, Special Issue on Polynomial Elimination 28 (1999), 125-154.

[2] Aubry, P., Lazard, D., and Moreno-Maza, M. On the theories of triangular sets. Journal of Symbolic Computation, Special Issue on Polynomial Elimination 28 (1999), 105-124.

[3] Collins, G. E. Quantifier elimination for real closed fields by cylindrical algebraic decomposition. $A u$ tomata theory and formal languages (Second GI Conf., Kaiserslautern, 1975), pp. 134-183. Lecture Notes in Comput. Sci., Vol. 33, Springer, Berlin, 1975.

[4] Faugère, J.-C. A new efficient algorithm for computing Gröbner bases (F4).-. Journal of Pure and Applied Algebra 139, 1-3 (1999), 61-88.

[5] Hong, Hoon. An efficient method for analyzing the topology of plane real algebraic curves. Symbolic computation, new trends and developments (Lille, 1993). Math. Comput. Simulation, 42 (1996), no. 4-6, 571-582.

[6] Kalkbrener, M. A generalized Euclidean algorithm for computing triangular representations of algebraic varieties J. Symbolic Comput.15 (1993) 143-167.

[7] Lazard, D. A new method for solving algebraic systems of positive dimension. Applied algebra, algebraic algorithms, and error-correcting codes (Toulouse, 1989). Discrete Appl. Math. 33 (1991), no. 1-3, 147-160.

[8] Lazard, D. Resolution of polynomial systems. Computers Mathematics. Proceedings of the Fourth Asian Symposium (ASCM 2000). Xiao-Shan Gao, Dongming Wang ed. World Scientific (2000), 1-8.

[9] Lu Yang and Zhenbing Zeng. Equi-Cevaline points on triangles. Computers Mathematics. Proceedings of the Fourth Asian Symposium (ASCM 2000). Xiao-Shan Gao, Dongming Wang ed. World Scientific (2000), 130-137.

[10] Rouillier, F. Solving zero-dimensional systems through the rational univariate representation. Appl. Algebra Engrg. Comm. Comput. 9 433-461 1999.

[11] Rouillier, F., and Zimmermann, P. em Efficient Isolation of a Polynomial Real Roots. Research report INRIA RR-4113, 16 p. (Feb. 2001). http://www.inria.fr/rrrt/rr-4113.html

[12] Wang, Dongming Elimination methods. Springer, Wien New York, 2000.

[13] Wu, Wen Jun. Basic principles of mechanical theorem proving in elementary geometries. J. Systems Sci. Math. Sci. 4 (1984), no. 3, 207-235. 